in terms of the established measure of selective information-rate. The interpretation of such experiments, and others of like kind, is that, under a considerable variety of error-making conditions, the rate of selective-information in the human 'black-box' channel remains substantially constant. Dr. Hick gave a number of illustrations of such experiments, involving stimuli and responses of several different forms, and of extensions of the method to apply to an alphabet of signal-stimuli having unequal (objective) probabilities of occurring. He afterwards discussed these experiments, and their interpretations by the selective-information-content measure, in relation to other studies of reactions, emphasizing at the same time that the common idea of 'reactiontime' as a kind of constant, so frequently employed in tests for drivers and air pilots, is grossly inadequate. Dr. Hick ended his lecture by emphasizing that his experiments consisted purely of observations of behaviour and that, where probabilities were mentioned, they were objective, relative frequencies; nevertheless, he suggested, information theory has further value for psychology in that it provides a number of general concepts and terms which may aid discussion, at the purely subjective levelsthough care must be taken to distinguish such subjective inquiry from direct, quantitative applications of information theory to behavioural psychology.

The last speaker, Dr. D. M. MacKay, distinguished three different ways in which brains are often compared with machines, for three different purposes. The first is the popular comparison between brains and existing machines, notably electronic digital computers. It is a relevant comment that such machines are deliberately designed not to imitate the brain in most of its more characteristic functions ; on the contrary, they may be said to take over some of the more machine-like functions of the mind. The second concerns the question as to whether we can ever design an artefact ('artificial mechanism') to imitate any specified aspect of human behaviour; for example, to frame novel hypotheses or (notoriously) to write sonnets.

But the third manner of brain/machine comparison to which Dr. MacKay referred really formed his main theme: Can we build a model working internally on the same principles as the brain ? Such may not be an actual engineered machine, but a conceptual model, conforming to all conditions demanded by present knowledge, broad though they be, and having as its purpose the setting up of a description not in the distinct languages of psychiatry, or physiology, or anatomy, but in language common to all these; to provide working links between these various fields. The language of information and control theory promises to be of value in this move toward unification of concepts because, in a sense, it belongs to both the psychology field and the domain of direct physical observation (physics, physiology ... 'mechanism'). The theoretical characteristics of such a model may then be checked against observation of the brain in action, the discrepancies yielding information towards refining or replacing the model; and so repeatedly, by the usual inductive-deductive procedure. It is important that such models, or descriptions, should not only 'work' in the manner of a normal brain, and meet all functional tests in a similar way, but also that they should 'go wrong' likewise - though Dr. MacKay stressed that this is very different from naïvely attributing mental illness to electronic computing machines that break down.
A further requirement of the model is that, like the brain, it must have been capable of 'growing that way'; such consideration has led to investigations of the possibilities of self-organizing statistical models-models in which the various elements not only are interconnected more or less randomly, but which also themselves function indeterministically to some controllable extent. Such a model should be capable of adjusting the rules of its activity according to the degree of success it attains, by adjusting the relative probabilities of different patterns of activity. Dr. MacKay elaborated upon such a model, describing how, given some positive indication of success or failure, it could continually improve its 'goal-seeking' activity, and "grope its way more and more quickly into a pattern of activity to 'match' any incoming patterns that persisted in occurring".

Finally, Dr. MacKay dealt with questions of purpose. Such theoretical models are intended, he stressed, as research tools. They represent a method of describing 'the thinking process' in disciplined language, suitably objective as to assist the physiologist, yet not divorced from psychiatric reality, so as to permit further assistance in diagnosis, study and treatment of mental illness.

Such work is so often misinterpreted as an attempt to depose man from his special position; man would be 'nothing but' a machine, we often hear, if the brain is described in physical terms. The fault with such reductionist argument, as Dr. MacKay stressed, lies not in the possible incompleteness of the description, but in confusion between two distinct languagesystems. Models of the brain form descriptions in observer-language-a set of directions by which, for example, replicas can be made ; but a man's personal experience, or rather his statements about his experience, are in actor-language. Questions of personal decision-making, or questions of responsibility, simply do not form part of the brain-model because they cannot be framed in observer-language. However great our understanding of brain structure and functioning, however accurate the model, we none of us are one jot relieved from responsibility for our actions.

The discussion which followed centred mainly around the different uses of the words 'model', 'artefact', or 'mechanism' in such contexts. In response to one speaker who asked if real working models had actually yet been made, Prof. Ross Ashby gave a brief account of his 'homeostat' model, and of its behaviour. E. Colin Cherry

\section{UTILIZATION OF DIGITAL COMPUTING MACHINES}

$\mathrm{A}^{\mathrm{T}}$

$T$ the recent meeting in Liverpool of the British Association, Section G (Engineering) held a symposium on the utilization of digital computing machines in engineering and industry. Prof. F. C. Williams, of the University of Manchester, opened the meeting. Before introducing the main speakers, he said that the object of the session was to strip the 'electronic brain' of some of its glamour and to present it to engineers in its everyday shape, which is that of a potentially powerful new tool to aid the solution of engineering design problems. Although at present this tool is in an early stage of development, it is none the less already capable of revolutionizing design procedures-for example, by the rapid 
analysis of a large number of trial designs of turbine shafts or steel frameworks.

Dealing with the machine at the University of Manchester, Prof. Williams said that it originated from the joint initiative of himself and Prof. M. H. A. Newman, of the Mathematics Department. Although the machine was originally built to gain experience in design techniques, it soon became apparent that it is capable of handling a volume of work far in excess of the University's needs, and for this reason it was made available to other universities and to industry. This use of the machine by outside organizations has increased rapidly and, what is perhaps more important, those organizations which have started to use the machine have found themselves calling upon its services to an increasing extent as their experience of its potentialities increases. The University does not undertake the solution of problems, but instead trains representatives of other organizations in the use of the machine and afterwards makes the machine available to them. The period of training is about two or three months.

On the question of reliability, Prof. Williams said that, at the present stage of development, machines are serviceable for about 80 per cent of the time, and this is quite sufficient to enable them to be used as the basis of a computing service run on business lines. As in the case of human computers working with desk machines, electronic machines are liable to make transient mistakes, and results should be checked, also with the machine, in the usual way. However, because of the high speed of the machine, a fault-free run of several minutes corresponds to less than one mistake per week by a human computer. Finally, Prof. Williams stressed the ease with which digital machines can be switched from one problem to another. It simply requires a new set of instructions, which can be 'fed' into the machine from punched tape or cards in a few seconds.

Prof. Williams then introduced the other speakers : Mr. R. K. Livesley, of the Computing Machine Laboratory, University of Manchester, and Mr. G. G. Alway, of the Mathematics Division of the National Physical Laboratory, Teddington.

Mr. Livesley described first the general principles on which electronic digital computers are based. The most important fact about these machines, he said, is that they carry out calculations according to a pre-arranged sequence of coded instructions. Once this sequence of instructions (known as the 'programme') has been prepared, the computer carries out the calculations automatically. It is the job of the machine user to prepare the programme for his particular problem, and this means that he has first. to decide in detail the sequence of arithmetic operations to be performed, and then translate this scheme into language understood by the machine.

It was emphasized by Mr. Livesley that, once the programme has been perfected, it can be used unchanged for different sets of numerical data. Thus a programme is very similar to a mass-production jig-both are capital investments which can be used an unlimited number of times. Like the machine tool to which the jig is attached, an electronic computer is most productive when engaged on repetition work-it is seldom worth while making either a jig or a programme if it is to be used only once. Such repetition work is very common in engineering calculations. Most of the numerical work associated with designing is concerned with the analysis of alternative layouts, the only difference in the calculations being the actual numbers occurring.

To illustrate the use of digital computers in design work, Mr. Livesley described four programmes developed for use with the Manchester computer. These cover the calculation of the natural frequencies of shaft systems in torsional and in lateral vibration, the design of certain types of cam, and the analysis of rigid-framed building structures. He said that the existence of these programmes means that certain common calculations can be carried out with scarcely any effort beyond that of feeding the initial data into the computer. On the Manchester machine it has been found that the speed factor over hand computation is often of the order of $150: 1$-an ample repayment for initial time spent on programming.

Mr. Livesley defined the ideal programme as one which carries out all the calculations of a problem, from the data on the designer's drawing-board to the final facts he wants to know, and which is sufficiently flexible to cover all problems of a given type. The first requirement helps to cut down possibility of error, while the second avoids the necessity of developing new programmes for very similar work. One usually finds that the human beings preparing data for a machine are far more prone to error than the machine itself, and consequently it is essential to make the input of data as simple as possible, getting the machine to do the easy parts of the calculation as well as the most difficult. The advent of com. puters, and the demand for flexibility in programmes, has led to interesting developments in numerical analysis. New methods, especially suited to automatic machines, have shown definite superiority when compared with methods generally in use on desk calculating machines.

At the present time, continued Mr. Livesley, there is a lack of programmes and programmers rather than of computing machines. It is only as programmes are developed for standard calculations that the existing machines can be fully used. The training time for a programmer is from three to twelve weeks, with a similar period for the time usually required to develop a programme for a given cal. culation. Mr. Livesley concluded by emphasizing that the efficient use of a computing machine depends entirely on the programmer.

The subject of Mr. Alway's paper was the application of the ACF, the machine at the National Physical Laboratory, Teddington, to aircraft design problems. Supplementing some remarks of Prof. Williams, Mr. Alway said that the ACE group also instructs people in the use of their machine, and, in addition, a large team of programmers enables them to undertake work directly. The pilot model of the ACE has been in operation for eighteen months, and during this time a considerable library of programmes has been built up. The machine has only a limited high-speed store; but the use of Hollerith punched cards as an auxiliary store has been very successful. Certain features of the logical design of the machine enable calculations to be done while cards are being read or punched. By this means the disparity between the speeds of operation of the auxiliary store and the working store is largely overcome.

The pilot model has been very successful in dealing with problems of matrix arithmetic. Programmes for solving sets of linear simultaneous equations have been made using the card store already described. 
These can solve sets involving up to 192 unknowns and can invert matrices of orders up to 95 . Programmes have also been made for finding the latent roots and vectors of matrices. A fast programme, in which all the numbers concerned are kept in the high-speed store, can deal with symmetric matrices of order 19 and unsymmetric matrices of order 15. For matrices of higher orders, punched cards are used as an auxiliary store. Similar programmes exist for dealing with complex latent roots. All these programmes are in constant use, and many applications have been made to problems arising in aircraft design.

Mr. Alway then described a typical problem of aerodynamic design, namely, the flutter problem. Flutter is the condition where the aerodynamic forces tend to reinforce the elastic forces in a vibrating wing, and the aeroplane must be designed so that this will not occur at any speed at which it is supposed to fly. The differential equations representing the complete motion of the whole aircraft as an elastic body subject to aerodynamic forces are intractable, but design engineers are helped if, as a first approximation, the modes of vibration of the aeroplane in vacuo are obtained. This involves finding the latent roots and vectors of a matrix. For the complete flutter problem the equations can be set up for a limited number of degrees of freedom, and their solution amounts to finding values of velocity and frequency which make a certain determinant zero. Each element of this determinant is of the form $a \omega^{2}+i b \omega+c+e / V^{2}+i d$, where $\omega$ is the frequency, $V$ the aircraft velocity, and $a, b$ and $c$ to some extent depend on $\omega$. One programme for dealing with this part of the flutter problem is arranged so that the complex determinant is evaluated for pairs of values of $\omega$ and $V$, and the values of $\omega$ and $V$ which make the determinant zero are then found by interpolation. The computation time for a single determinant of order 8 , corresponding to 8 degrees of freedom, is a quarter of a minute.

In conclusion, Mr. Alway pointed out that, unlike analogue simulators, an automatic digital computer becomes more powerful as more programmes are developed and existing techniques stream-lined.

\section{THE PHARMACIST AND THERAPEUTICS}

$\mathrm{T}$ HE British Pharmaceutical Conference, held during August 31--September 4, looked back through ninety years to its foundation in 1863, and its chairman this year, Dr. G. R. Boyes, contributed to it a valuable address entitled "The Pharmacist and Therapeuties". Three years before the long series of these conferences began, Oliver Wendell Holmes stated his belief that, if the whole of the materia medica then in use in the United States could have been sunk to the bottom of the sea, it would have been "all the better for mankind and all the worse for the fishes"; and Dr. Boyes considers that these words could have been applied also to the British materia medica of that day. The publication of the first British Pharmacopœia was a major step towards the improvement of British pharmacy, and the records of successive British Pharmaceutical Conferences show how much the pharmacists have contributed to the subsequent improvement in the methods of preparation, formulation and analytical control of substances used for the treatment and control of disease. Dr. Boyes, in his address, reviewed some of the many important contributions made by pharmacy to the materia medica of to-day.

The primary functions of a pharmacist are to provide the drugs and preparations of drugs needed by medical men, to see that these are pure and active and that they are supplied in forms suitable for the various methods of administration of them. The pharmacist thus bears a great responsibility and must have a thorough knowledge of the chemistry and physics of drugs, of analytical methods used for their assay and of the principles of drug formulation. $\mathrm{He}_{\Theta}$ needs also a knowledge of physiology and pharmacology. The 'chemist', in other words, from whom we buy our pills and potions is highly skilled in his jobs and is carefully trained for it. He has, however, fewer opportunities nowadays to exercise his skill in the art of compounding medicines, and more and more is he asked to supply medicaments which reach him ready for use.

Outstanding features of modern pharmacy are the extensive use of synthetic substances and the decline in the use of crude preparations, for which isolated substances active in them have been substituted. Synthetic substances were introduced as long ago as 1896 ; but their use has greatly increased during the past ten or twenty years, and Dr. Boyes quoted figures which indicate the astonishing growth and magnitude of the industry now engaged in the manufacture of these synthetic substances and isolated active principles. He rightly emphasized the value of the contributions made by pharmacy to this remarkable development.

The discovery of a new drug, whether it is used by medica? men or veterinarians, is seldom due to chance. It is usually the result of long periods of co-ordinated research, the testing of scores or hundreds of synthetic compounds, and the choice, as a result of all this work, of a few substances which promise to give the required balance between efficacy, ease of administration and a minimum of cost and of toxicity to the patient. The public are seldom fully aware of the amount of work of this kind involved, or of the degree of skill, experience and training needed for it. As Dr. Boyes says, work of this kind has developed for us a variety of drugs in use to-day, among which are the derivatives of barbituric acid, synthetic œstrogens and other sex hormones, sulphonamides, antimalarial drugs and drugs which reduce pain or induce anæsthesia.

At the same time, drugs derived from plants and animals have not been neglected. Dr. Boyes tells us of the work done on some of these. Among the plant-products are khellin, quinine, digitalis and morphine. The laboratory synthesis of morphine has recently been accomplished - a feat which only the chemist can rightly assess. Other plant-products are penicillin, streptomycin, aureomycin and the other antibiotics, to which new ones are constantly being added. Among animal products are the various. hormones, the substances used for blood-transfusions: and antitoxins. Dr. Boyes gave special attention to. the work on corticotrophin, insulin and the recent. isolation by chromatographic methods from raw liver of cyanocobalamin, which is also produced by Streptomyces griseus, the organism which yields streptomycin.

The remainder of Dr. Boyes's address was devoted to sections on the analytical control of drugs and to their biological and clinical trials. This part of 